\title{
Análisis de la automedicación como problema de salud
}

\author{
Claudia Sánchez Bermúdez,*Ma. Guadalupe Nava Galán**
}

\begin{abstract}
RESUMEN
En México son pocos los estudios o publicaciones que permiten valorar la real magnitud del consumo de medicamentos, hierbas y remedios caseros que se consumen por consejo de otra persona o por iniciativa propia, sin consultar al médico; es un fenómeno que se ha incrementando a través del tiempo, convirtiéndose en un serio problema de salud pública; esta práctica se presenta en la población del equipo de salud y cada vez es más común.
\end{abstract}

Palabras clave: Automedicación, enfermería, problema de salud.

\section{The self-medication health problem}

\begin{abstract}
In Mexico there are few studies or publications to assess the true magnitude of the consumption of drugs, herbs and home remedies that are consumed on the advice of another person or on its own, without consulting a doctor; is considered a phenomenon that has been increasing at over time, becoming a serious public health problem; this practice occurs in the population and the health care team is increasingly common.
\end{abstract}

Key words: Self-medication, nursing, health problem.

\section{INTRODUCCIÓN}

$\mathrm{L}$ a automedicación es definida como la ingestión de medicamentos de venta libre o por iniciativa propia. ${ }^{1}$ Actualmente es un problema que se vive en los países en vías de desarrollo como México. Quienes la llevan a cabo con más frecuencia son personal del equipo de salud ya que al conocer la patología y saber de farmacología tienden a realizar esta práctica por decisión propia o por sugerencia de amigos. A diferencia de México, la automedicación ha sido motivo de diferentes investigaciones en algunos países de América Latina; es una forma común de "autocuidado de la salud", principalmente en el equipo médico y paramédico, quienes están conscientes del consumo de producto que alivien síntomas de malestar general.

En ese mismo sentido, en el 2002 Burtica determinó la frecuencia y las características de la automedicación en estudiantes de la Universidad de Caldas, Colombia; demostró que el $47 \%$ se automedican; el $57.4 \%$ son mujeres y el $42.6 \%$ hombres; el $74 \%$ del total de los estudiantes son de estrato socioeconómico medio. La Universidad cuenta con varias facultades y los que más se automedican pertenecen a la Facultad de Ciencias para la Salud, con un 30.3\%. Los medicamentos más utilizados son antiinflamatorios no esteroideos $(67 \%)$. Los motivos: cefalea (33\%) y otros dolores $(22.4 \%) \operatorname{con}(\mathrm{p}<0.05){ }^{2}$

* Licenciada en Enfermería.

*** Maestra en Enfermería en Educación.

Correspondencia: Claudia Sánchez Bermúdez, Insurgentes Sur Núm. 3877, Col. La Fama, Deleg. Tlalpan, 14269, México, D.F. E-mail: rev. enf.neurol@gmail.com 
Así mismo, Alvitres llevó a cabo en el Hospital Público de San Salvador de Jujuy, Argentina, en el 2002, una investigación sobre autodiagnóstico (AD) y automedicación (AM) en el personal de enfermería, práctica usual en nuestros días. Refiere que el autodiagnóstico es el reconocimiento que se realiza por sí mismo de una enfermedad y la automedicación el posterior uso de medicamentos por iniciativa propia. Aún se discuten las causas que inciden en el autodiagnóstico y la automedicación del personal de enfermería (PE). Los resultados mostraron que el $71 \%$ de los profesionales de enfermería se autodiagnostican y automedican; los factores que influyen son: el económico, el medioambiental y el sociocultural; los medicamentos más utilizados son: analgésicos, antiinflamatorios, antipiréticos y antibióticos; se concluyó que es necesario concientizar al equipo de enfermería hacia cambios de conducta en el autoconsumo de medicamentos y en la revalorización del autocuidado de su salud (AU). ${ }^{3}$

En este orden de ideas, en Caracas, durante 2004, Madaldi investigó sobre farmacovigilancia y hábitos de consumo de medicamentos en 657 estudiantes de enfermería de la Universidad Central de Venezuela del Centro de Vigilancia Farmacológica. Se determinaron eventos adversos (EA) frecuentes asociados al uso de fármacos; se evaluaron hábitos de consumo de medicamentos, grupos farmacológicos de mayor uso, la automedicación y la preferencia de compra ante los medicamentos de marca registrada versus los genéricos. Los resultados revelaron que el $68.9 \%$ de los encuestados han experimentado algún evento adverso relacionado al consumo de medicamentos, siendo de tipo alérgico en un $8.9 \%$. En cuanto al hábito de consumo de fármacos, el $81 \%$ de los estudiantes practica la automedicación y el $78 \%$ prefiere consumir genéricos, ya que son económicos, en contraste con los de marca registrada. Se concluyó que la automedicación está muy extendida entre los estudiantes encuestados, corriendo el riesgo que representan los eventos adversos y el uso inadecuado de los fármacos. ${ }^{4}$

Con referencia a lo anterior, Aguado, en 2005, mostró lo usual que es la automedicación en estudiantes de Farmacia de la Universidad Nacional del Nordeste (UNNE), en Argentina, ya que la población seleccionada conoce los fármacos y los tienen al alcance. Los alumnos evaluados son de la Carrera de Farmacia, de la Facultad de Agroindustrias (Universidad Nacional del Nordeste) de Sáenz Peña, Chaco; la muestra fue del $51.8 \%$ de la población (216 estudiantes); se definió el perfil de conducta de los estudiantes; los resultados permitieron establecer algunos parámetros sociosanitarios para disminuir los riesgos de la medicación. ${ }^{5}$

Respecto a lo anterior, Grela A en el 2006, en Argentina, determinó la prevalencia de la conducta de automedicación en estudiantes del 5 o año de la Facultad de Medicina de la
Universidad Nacional del Nordeste para establecer cuáles son los medicamentos más consumidos en la automedicación e identificó qué los motiva para llevarla a cabo; también las fuentes de información consultadas para desarrollar esta conducta. Los resultados determinaron que el $92 \%$ de los alumnos de 50 año han empleado uno o más medicamentos por cuenta propia, sin prescripción médica en los últimos seis meses. ${ }^{6}$

Los más utilizados: analgésicos, antiinflamatorios y antifebriles en un $84 \%$. El principal motivo, en el 50\%, es la presencia de síntomas leves; un 40\% recibieron información de una prescripción anterior y un elevado porcentaje de alumnos incurrió en esta práctica, coincidiendo con otros estudios; los medicamentos más utilizados por la población son los analgésicos; debido a la sintomatología, los antibióticos ocupan el $2^{\circ}$ lugar, representando un alto riesgo por favorecer la aparición de cepas resistentes; se concluye que los alumnos de medicina presentan un elevado porcentaje de automedicación, a pesar de conocer el alto riesgo que dicha conducta representa.

En Brasil (2006), Tizón Bouza investigó si se automedica un grupo de profesionales de enfermería de atención hospitalaria e identificaron los medicamentos más empleados y los medios para obtenerlos. Los sujetos de estudio fueron enfermeras y auxiliares de Enfermería del Complejo Hospitalario Arquitecto Marcide-Profesor Novoa Santos, de A Coruña; además, se recabó información sobre la frecuencia de consumo, tipo de medicamentos, prescripción y forma de obtención, motivos para automedicarse y opinión acerca de la práctica de la automedicación, entre otras variables. Los resultados obtenidos: 91\% (51) de las profesionales de enfermería encuestadas tomaba medicamentos regularmente y sólo en el $14.3 \%$ (8) eran prescritos; apenas el 8.9\% (5) acude siempre a su médico de cabecera para diagnóstico y prescripción.?

Entre las patologías que destacaron para automedicarse se reportaron: cefalea, dolor de espalda, fiebre o traumatismos; los fármacos más consumidos sin prescripción médica fueron antiinflamatorios/analgésicos, antipiréticos y antigripales, por lo cual se llegó a la conclusión de que la prevalencia de automedicación es de un $85.6 \%$, lo que coincide con la opinión de las enfermeras a favor de una automedicación responsable para procesos leves.

Con referencia a lo anterior, Rocha Barros en el 2009 investigó la prevalencia de la automedicación y los factores asociados entre profesionales de enfermería; se realizó un estudio epidemiológico seccional que abarcó 1,509 enfermeros de dos hospitales públicos en Río de Janeiro, Brasil. Los medicamentos fueron identificados y clasificados de acuerdo con el Anatomical Therapeutic Chemical Index. La prevalencia de automedicación fue de $24.2 \%$; el grupo 
anatómico más referido fue el sistema nervioso y el grupo terapéutico incluyó los analgésicos.

La prevalencia fue más alta entre los más jóvenes, en aquéllos con disturbios psíquicos menores, los no hipertensos, los que no hacían ejercicio físico, los que refirieron enfermedad o herida en los últimos 15 días y en aquéllos con mayor número de enfermedades autodiagnosticadas. Los enfermeros de contrato temporal y los que refieren doble jornada de trabajo practican la automedicación con frecuencia, lo cual está asociado a diversos factores que deberían ser considerados como estrategias que buscan mejores condiciones de salud entre ellos. ${ }^{8}$

Como se ha mencionado, en la ciudad de Corrientes, Argentina, Aráoz Olivos publicó en 2009 un análisis donde abordaba la automedicación en 182 estudiantes del internado rotatorio de la Facultad de Medicina y para determinar, entre otras cosas, si existía una actitud favorable o desfavorable. La muestra fue aleatoriamente analizada: sexo, edad, recomendación del consumo, tipo de medicamento y efectos adversos. Se encontró que $48.25 \%$ correspondía al sexo femenino y $51.75 \%$ al masculino. La edad media fue de 24 años, $76.71 \%$ se automedican por conocimientos propios, $10.96 \%$ por farmacéutico, $10.96 \%$ por amigos o familiares y $1.37 \%$ por publicidad. El tipo de medicamento más usado en $41.98 \%$ son los antiinflamatorios no esteroideos (AINES), $16.41 \%$ antibióticos, $11.07 \%$ anticonceptivos, $9.54 \%$ antialérgicos, $6.87 \%$ antiácidos, $5.34 \%$ antimigrañosos, $4.58 \%$ corticoides, $2.30 \%$ psicofármacos y $0.38 \%$ laxantes. Del total, $20.28 \%$ presentaron efectos adversos con automedicación, siendo gastritis $44.82 \%$, diarrea $37.93 \%$, náuseas/ vómitos $27.58 \%$, alergias $20.68 \%$, cefaleas/mareos $3.44 \%$. $^{9}$ Se publicó en la Revista Mexicana de Ciencias Farmacéuticas un estudio de automedicación en una farmacia comunitaria de la ciudad de Toluca para determinar la frecuencia y los factores que predisponen a la automedicación. El instrumento de recolección de datos fue un cuestionario que pedía información sociodemográfica, económica, cobertura del sistema sanitario y de automedicación. Los resultados mostraron un mayor porcentaje de automedicación en mujeres de población adulta y en sujetos con menor acceso a servicios sanitarios. Los medicamentos que más se consumieron fueron: analgésicos, antibióticos y antiácidos. Con este estudio se determinaron los grupos prioritarios a los que se deben dirigir programas de educación sanitaria, para mejorar la calidad de la automedicación. ${ }^{10}$

Guillem Sáiz, en 2010, publicó en la Revista Clínica de Medicina Familiar el estudio Automedicación en Población Universitaria Española; su objetivo fue determinar si la automedicación es una práctica común en diferentes sociedades y qué tanto puede tener importantes implicaciones sanitarias, además de cuantificar esta actividad para estudiar los factores asociados a la misma. Esta práctica ha sido poco estudiada hasta ahora en población universitaria española. Se evaluó sexo, estudios sanitarios y hábitos de información sobre medicamentos. Los resultados de la práctica de automedicación fueron confirmados en el $90.8 \%$ de las 501 personas que contestaron correctamente la encuesta. Esta práctica es significativa en mujeres; los medicamentos más consumidos son analgésicos/antiinflamatorios con un 73\%. Las mujeres consumen más anticatarrales, mientras los hombres más antibióticos. Los individuos con hábitos de información sobre medicamentos presentaron un menor consumo de antibióticos. Se pudo concluir que la práctica de la automedicación es elevada en población universitaria valenciana y mayor de la reportada anteriormente en nuestro país. El sexo y los hábitos de información están asociados a la automedicación en determinados grupos de medicamentos. ${ }^{11}$

\section{CONCLUSIÓN}

Se concluye de este análisis que la automedicación es ejercida indiscriminadamente por parte de los estudiantes. Los medicamentos más utilizados fueron antiinflamatorios no esteroideos y antibióticos. Los efectos adversos correspondieron al aparato gastrointestinal por no considerar los efectos adversos y el seguimiento del tratamiento. Se utilizan medicamentos por conocimientos propios ya que la adquisición es de venta libre y por lo mismo incita a hacerlo sin mostrar alguna diferencia con la autoprescripción.

La automedicación constituye una práctica riesgosa cuyas consecuencias pueden ser, entre otras: enmascaramiento de una enfermedad y retraso en la asistencia médica en casos en los que es realmente necesaria: aparición de reacciones adversas e interacciones medicamentosas, aumento de la resistencia a agentes antimicrobianos, elección incorrecta de medicamentos y riesgo de abuso o farmacodependencia ya que un medicamento es una sustancia con propiedades para el tratamiento o la prevención de enfermedades en los seres humanos y si no se lleva un control adecuado puede llevar a una dependencia sin darse cuenta.

\section{BIBLIOGRAFÍA}

1. Rocha A. Automedicación entre los trabajadores de enfermería de hospitales públicos. Rev Latino-Am Enfermagem 2009; 17 (6): Disponible en: http://www.scielo.br/scielo.php?pid=S010411692009000600014\&s cript=sci_arttext\&tlng=es (Acceso: 10 de octubre del 2010).

2. Burtica O. Automedicación en los estudiantes de la Universidad de Caldas. Rev Ciencias Básicas Biosalud. Disponible: http://biosalud.ucaldas. edu.co/downloads/Revista\%202_2.pdf (Acceso: 11 de octubre del 2010).

3. Alvitres B. Autodiagnóstico y automedicación en el personal de enfermería: una práctica usual en nuestros días. Rev Saude 2002; 10 (49): 37-40. Disponible en: http://bases.bireme.br/cgibin/wxislind.exe/iah/ 
online/?IsisScript $=$ iah/iah $. x i s \& s r c=$ google $\&$ base $=$ LILACS $\& l a n g=p \&$ nextAction=lnk\&exprSearch=324245\&indexSearch=ID (Acceso: 15 de octubre del 2010).

4. Magaldi L, Rocafull J. Farmacovigilancia y hábitos de consumo de medicamentos en los estudiantes de la Escuela de Enfermería de la Universidad Central de Venezuela. Rev Facultad de Medicina 2004; 27 (1): 74-78. ISSN 0798-0469. Disponible en: http://www.scielo.org.ve/ scielo.php?pid=S0798- (Acceso: 20 de Octubre del 2010).

5. Aguado M. Automedicación en Estudiantes de Farmacia de la Universidad Nacional del Nordeste. Rev Acta Farm Bonaerense 2005; 24 (2): 271-6. Disponible en: http://www.latamjpharm.org/trabajos/24/2/ LAJOP_24_2_5_3_WA44D3E949.pdf (Acceso: 21 de octubre del 2010).

6. Grela A. Automedicación: Experiencia en estudiantes de $5^{\circ}$ año de la Facultad de Medicina de la Universidad Nacional del Nordeste. Rev de Postgrado de la VIa Cátedra de Medicina - No. 155-Marzo 2006. Disponible en: http://www.med.unne.edu.ar/revista/revista155/2_155. pdf (Acceso: 22 de octubre del 2010).
7. Tizón E. Automedicación en el personal de enfermería hospitalaria. Rev Enferm Clin 2006; 16: 210-3. Disponible en: http://dialnet.unirioja.es/ servlet/articulo?codigo=2037354 (Acceso: 22 de octubre del 2010).

8. Barros JAC. Políticas farmacéuticas: a servicio dos interesses da saúde? Brasília: UNESCO. 2004. Disponible en: http://unesdoc.unesco.org/ images/0013/001360/136090por.pdf (Acceso: 20 de octubre del 2010).

9. Aráoz N. La automedicación en estudiantes del internado rotatorio. Revista de Posgrado de la Vía Cátedra de Medicina. No. 197 - Enero 2010. Disponible en: http://www.med.unne.edu.ar/revista/revista197/1_197.pdf (Acceso: 22 de octubre del 2010).

10. Gómez OML. Estudio de automedicación en una farmacia comunitaria de la ciudad de Toluca. Rev Mexicana de Ciencias Farmacéuticas 2009 40 (1): 5-1. Disponible en: http://redalyc.uaemex.mx/pdf/579/57940102. pdf (Acceso: 22 de octubre del 2010).

11. Guillem P. Automedicación en Población Universitaria Española. Rev Clin Med Fam 2010; 3 (2): 99-103. Disponible en: http://scielo.isciii. es/scielo.php?pid=S1699 695X2010000200008\&script=sci_abstract (Acceso: 24 de octubre del 2010). 